\title{
Adapted Fuzzy Integral: An Application in the Finite Element Method
}

\author{
D. SÁNCHEZ ${ }^{1,2 *}$, L.T. BASSANI ${ }^{3}$, L.C. BARROS ${ }^{1}$ and E. ESMI ${ }^{1}$ \\ Received on March 28, 2017 / Accepted on January 22, 2018
}

\begin{abstract}
In this paper we study and define an adapted fuzzy integral, based on the Sugeno integral. Moreover, we present a numerical integration formula which approximates the value of the adapted fuzzy integral. Thus, we prove that the Riemann integral and the adapted fuzzy integral are equivalent for power functions. Next, we apply the formula proposed in the numerical integration, required in the finite element method, to obtain a numerical solution of a boundary value problem for the one-dimensional Poisson equation. Finally, we observed better results of the approximate solution obtained in the example with the use of our formula when compared with the simple trapezoidal rule.
\end{abstract}

Keywords: Fuzzy Measure, Sugeno Integral, Finite Element Method, Boundary Value Problem.

\section{INTRODUCTION}

The finite element method (FEM) is a general tool to obtain numerical solutions to differential equations. This method is used in various fields of knowledge, such as mathematics, physics and engineering, and in several applications modeled by boundary value problems (BVPs) [11].

The Sugeno integral was introduced in 1974 for functions whose co-domain is the interval $[0,1]$, that is, it was created to deal with membership functions of fuzzy subsets [12].

However, analytical or numerical integration of functions, that may not represent fuzzy subsets, are required in FEM to solve a BVP. Here, we introduce a numerical approximation for an adapted fuzzy integration based on the Sugeno integral.

This paper is organized as follows. Section 2 introduces some concepts of fuzzy sets theory. In Section 3, we briefly present the FEM to solve a BVP (in a classical way). In Section 4, we define an adapted fuzzy integral based on the Sugeno integral and we propose a numerical integration formula for monotonic and differentiable functions whose range is $[0, k]$, where $k \in$

\footnotetext{
*Corresponding author: Daniel Sánchez - E-mail: daniel@ime.unicamp.br.

${ }^{1}$ Departamento de Matemática Aplicada, IMECC, Universidade Estadual de Campinas, R. Sérgio Buarque de Holanda, 651, 13083-859, Campinas, SP, Brasil. E-mail: laeciocb@ime.unicamp.br; eelaureano@ime.unicamp.br ${ }^{2}$ Campus Patagonia, Universidad Austral de Chile, Coyhaique, Chile.

3 Departamento de Matemática Aplicada, IME, Universidade de São Paulo, R. Matão, 1010, 05508-090, São Paulo, SP, Brasil. E-mail: lbassani@ime.usp.br
} 
$\mathbb{R}^{+}$. In Section 5 we show the approximate resolution of a BVP using our approach and we compare these results with the ones obtained by employing the well-known trapezoidal rule. Finally, in Section 6, we conclude with some final remarks.

\section{BASIC CONCEPTS OF FUZZY SETS THEORY}

Definition 2.1 (Fuzzy subset). [3, 4, 13] A fuzzy subset A of a (classical set or) universal set $\mathbb{U}$ is characterized by its membership function

$$
\phi_{A}: \mathbb{U} \rightarrow[0,1]
$$

If the range of the function $\phi_{A}$ is the set $\{0,1\}$ in (2.1), then $A$ is said to be a crisp subset.

Definition 2.2 ( $\alpha$-level). Let $A$ be a fuzzy subset of $\mathbb{U}$ and $\alpha \in[0,1]$. The $\alpha$-level of the fuzzy subset $A$ is the classical subset of $\mathbb{U}$ defined by

$$
[A]_{\alpha}=\left\{x \in \mathbb{U}: \phi_{A}(x) \geq \alpha\right\}, \text { for } \alpha \in[0,1] .
$$

Unless otherwise stated, we focus on the fuzzy subsets $A$ of $\mathbb{R}$, such that their $\alpha$-levels are given by $[A]_{\alpha}=\left[a_{\alpha}^{-}, a_{\alpha}^{+}\right], a_{\alpha}^{-} \leq a_{\alpha}^{+}$, or $[A]_{\alpha}=\varnothing$ for each $\alpha \in(0,1]$. We use the symbol $\mathbb{R}_{\alpha}$ to denote the class of these fuzzy subsets.

Definition 2.3 (Fuzzy measure). [1,8] Let $\mathscr{A}$ be a $\sigma$-algebra of a (classical) set $\Omega$. A map $\mu: \mathscr{A} \rightarrow[0, \infty)$ is called a fuzzy measure when it satisfies:

i) $\mu(\varnothing)=0$ and

ii) if $A, B \in \mathscr{A}$ and $A \subseteq B$, then $\mu(A) \leq \mu(B)$.

The definition of fuzzy measure proposed by Sugeno in [12] adds the boundary condition $\mu(\Omega)=$ 1 in $i$ ), that is, a normalization of the fuzzy measure, which implies that $\mu$ is a function from $\mathscr{A}$ to $[0,1][1]$. For this work, we denote $\widetilde{\mu}$ to the normalized fuzzy measures.

Definition 2.4 (Usual Lebesgue measure). Let $A \in \mathbb{R}_{\alpha}$ and $\alpha \in[0,1]$. The usual Lebesgue measure $\mu$ of the $\alpha$-level of $A$ is given by

$$
\mu\left([A]_{\alpha}\right)=a_{\alpha}^{+}-a_{\alpha}^{-}
$$

The usual Lebesgue measure $\mu$ is a fuzzy measure [3].

\section{GALERKIN FINITE ELEMENT METHOD}

Here, let us consider a linear boundary value problem (BVP) with homogeneous Dirichlet boundary values, in $x \in[0,1]$, that describes Poisson's one-dimensional equation given by [11]:

$$
\left\{\begin{array}{l}
-\frac{d^{2} u}{d x^{2}}=f(x), \quad 0<x<1 \\
u(0)=0, \quad u(1)=0
\end{array}\right.
$$


Using the traditional way to find an approximate solution for this BVP by FEM, we multiply the differential equation by test functions $v \in V^{0}$, where $V^{0}$ is a Hilbert space that considers the boundary values and is defined by [2]

$$
V^{0}=\left\{v \in C^{1}[0,1]: \int_{0}^{1}\left(v(x)^{2}+v^{\prime}(x)^{2}\right) d x<\infty, v(0)=v(1)=0\right\},
$$

considering the weak derivative for $v(x)$.

In this context, we can find $u \in V^{0}$ such that

$$
-\int_{0}^{1} u^{\prime \prime}(x) v(x) d x=\int_{0}^{1} f(x) v(x) d x, \forall v \in V^{0},
$$

and using the equivalent of Riemann's integration by parts we obtain that

$$
-\int_{0}^{1} u^{\prime \prime}(x) v(x) d x=\int_{0}^{1} u^{\prime}(x) v^{\prime}(x) d x-\left[u^{\prime}(x) v(x)\right]_{0}^{1}=\int_{0}^{1} u^{\prime}(x) v^{\prime}(x) d x .
$$

Thus, we obtain the variational formulation [2] for problem (3.1), which consists of determining $u \in V^{0}$ that satisfies

$$
\int_{0}^{1} u^{\prime}(x) v^{\prime}(x) d x=\int_{0}^{1} f(x) v(x) d x, \forall v \in V^{0} .
$$

Solving the BVP (3.1) is equivalent to solving a problem in a variational formulation, according to equation (3.2) [2].

Since $u \in V^{0}$ (and $V^{0}$ is an infinite dimensional Hilbert space), we want to build an approximate solution $u_{h}$ on a finite dimensional subspace $V_{h}^{0}$ of $V_{h}$ [6]. To this end, we consider a partition of the interval $[0,1]$ :

$$
\tau_{h}: 0=x_{0}<x_{1}<\cdots<x_{n}<x_{n+1}=1
$$

where $h=x_{i}-x_{i-1}, i=1,2, \ldots, n+1$. In this work, we use the finite dimensional vector space given by:

$$
V_{h}^{0}:=\left\{v_{h}: v_{h} \text { is a piecewise linear and continuous function on } \tau_{h}, v_{h}(0)=v_{h}(1)=0\right\} .
$$

Moreover, we consider the hat-functions (see Figure 1) as the piecewise linear basis functions $\left\{\varphi_{i}\right\}_{i=1}^{n}$ given by

$$
\varphi_{i}(x)=\left\{\begin{array}{cc}
\frac{x-x_{i-1}}{h}, & x_{i-1} \leq x \leq x_{i} \\
\frac{x_{i+1}-x}{h}, & x_{i} \leq x \leq x_{i+1} \\
0, & \text { otherwise }
\end{array}\right.
$$

Using this finite element formulation (or this discrete variational formulation) [2, 6], approximating the solution of (3.1) consists in obtaining $u_{h} \in V_{h}^{0}$, such that

$$
\int_{0}^{1} u_{h}^{\prime}(x) v^{\prime}(x) d x=\int_{0}^{1} f(x) v(x) d x, \forall v \in V_{h}^{0} .
$$




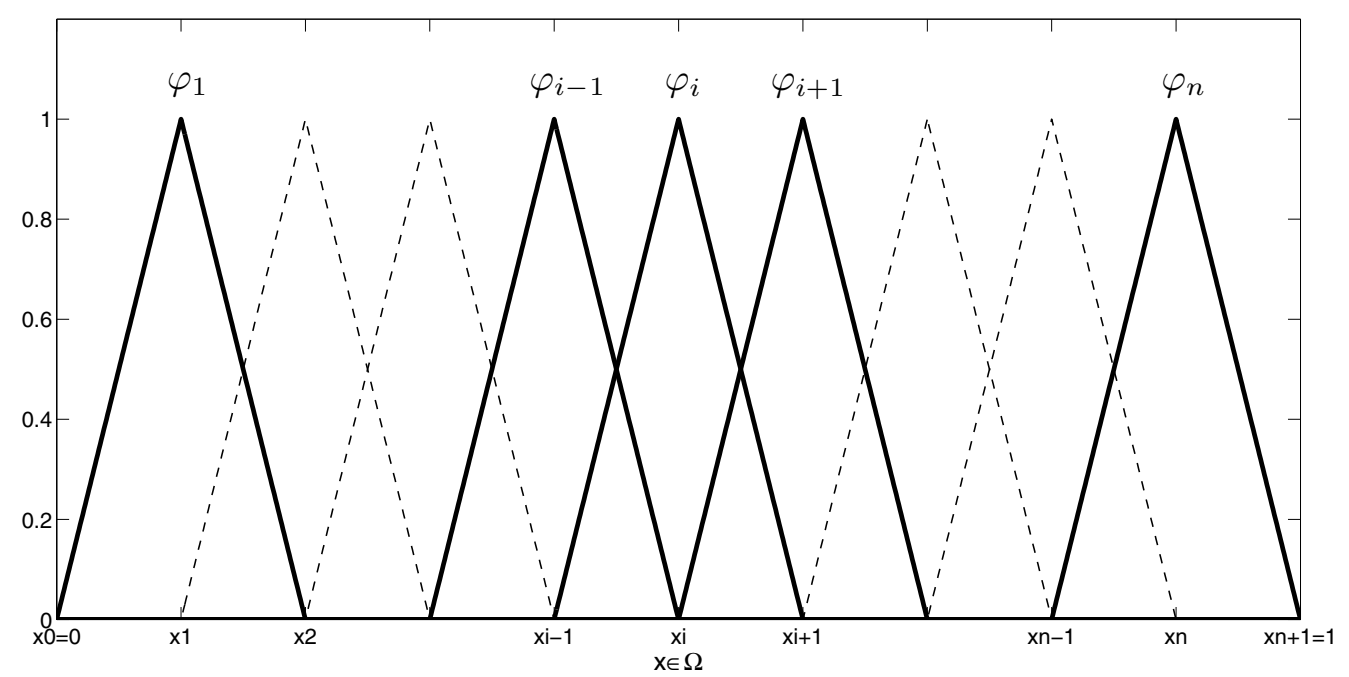

Figure 1: Piecewise linear basis functions of $V_{h}^{0}$.

Using the basis functions of $V_{h}^{0}\left(\varphi_{j} \in V_{h}^{0}\right), u_{h}$ is given by a linear combination of functions $\varphi_{j}$ $[2,6]$, with coefficients $\xi_{j}$ such that

$$
u_{h}(x)=\sum_{j=1}^{n} \xi_{j} \varphi_{j}(x) \quad \text { and } \quad u_{h}^{\prime}(x)=\sum_{j=1}^{n} \xi_{j} \varphi_{j}^{\prime}(x) .
$$

Since $u_{h}(x)$ in (3.4) is an approximation to $u(x)$, we have

$$
\sum_{j=1}^{n} \xi_{j}\left(\int_{0}^{1} \varphi_{j}^{\prime}(x) v^{\prime}(x) d x\right)=\int_{0}^{1} f(x) v(x) d x, \forall v \in V_{h}^{0} .
$$

Taking $v(x)=\varphi_{i}(x)$, for each $i=1,2, . ., n$, we can find $\xi_{j}$, for $j=1,2, \ldots, n$, by solving the following system of linear equations

$$
\sum_{j=1}^{n}\left(\int_{0}^{1} \varphi_{j}^{\prime}(x) \varphi_{i}^{\prime}(x) d x\right) \xi_{j}=\int_{0}^{1} f(x) \varphi_{i}(x) d x, i=1,2, \ldots, n .
$$

Thus, we have a discrete problem represented by a linear system of equations $[2,6]$, that can be given in the matrix form as

$$
A \xi=\mathbf{b}
$$

where $A=\left[a_{i j}\right]_{i, j=1}^{n} \in \mathbb{R}^{n \times n}$, with

$$
a_{i j}=\int_{0}^{1} \varphi_{i}^{\prime}(x) \varphi_{j}^{\prime}(x) d x
$$


$\xi=\left[\xi_{j}\right]_{j=1}^{n} \in \mathbb{R}^{n}$, and $\mathbf{b}=\left[b_{i}\right]_{i=1}^{n} \in \mathbb{R}^{n}$, with

$$
b_{i}=\int_{0}^{1} f(x) \varphi_{i}(x) d x
$$

If we assume the basis functions (3.3), then $\left\{\varphi_{i}^{\prime}\right\}_{i=1}^{n}$ are defined, for $i=1,2, \ldots, n$, as follows:

$$
\varphi_{i}^{\prime}(x)=\left\{\begin{array}{cc}
\frac{1}{h}, & x_{i-1} \leq x \leq x_{i} \\
-\frac{1}{h}, & x_{i} \leq x \leq x_{i+1} \\
0, & \text { otherwise }
\end{array}\right.
$$

Thus, the coefficients of matrix $A$, described above in (3.6), are given by

$$
a_{i i}=\int_{0}^{1} \varphi_{i}^{\prime}(x) \varphi_{i}^{\prime}(x) d x=\int_{x_{i-1}}^{x_{i}}\left(\frac{1}{h} \cdot \frac{1}{h}\right) d x+\int_{x_{i}}^{x_{i+1}}\left(\frac{-1}{h} \cdot \frac{-1}{h}\right) d x=\frac{2}{h},
$$

for $i=1,2, \ldots, n$, and

$$
a_{i, i-1}=\int_{0}^{1} \varphi_{i}^{\prime}(x) \varphi_{i-1}^{\prime}(x) d x=\int_{x_{i-1}}^{x_{i}}\left(\frac{1}{h} \cdot \frac{-1}{h}\right) d x=-\frac{1}{h},
$$

for $i=2,3, \ldots, n$.

Since $A$ is symmetric, we have that $a_{i-1, i}=a_{i, i-1}$ and by the definition of the basis we conclude that $a_{i j}=0$ for $|i-j|>1$.

Thus, we may obtain vector $\mathbf{b}$ in (3.7), by using the simple trapezoidal rule to approximate a numerical integration:

$$
\begin{aligned}
b_{i} & =\int_{0}^{1} f(x) \varphi_{i}(x) d x=\int_{x_{i-1}}^{x_{i}} f(x) \varphi_{i}(x) d x+\int_{x_{i}}^{x_{i+1}} f(x) \varphi_{i}(x) d x \\
& =\int_{x_{i-1}}^{x_{i}} f(x) \frac{x-x_{i-1}}{h} d x+\int_{x_{i}}^{x_{i+1}} f(x) \frac{x_{i+1}-x}{h} d x \\
& \approx \frac{x_{i}-x_{i-1}}{2} f\left(x_{i}\right)+\frac{x_{i+1}-x_{i}}{2} f\left(x_{i}\right)=h f\left(x_{i}\right) .
\end{aligned}
$$

Thus, if $f\left(x_{i}\right)=f_{i}$ for $i=1,2, \ldots, n$, then the final configuration of system (3.5) is

$$
A=\frac{1}{h}\left[\begin{array}{ccccc}
2 & -1 & 0 & \cdots & 0 \\
-1 & 2 & -1 & \ddots & \vdots \\
0 & -1 & 2 & \ddots & 0 \\
\vdots & \ddots & \ddots & \ddots & -1 \\
0 & \cdots & 0 & -1 & 2
\end{array}\right], \quad \xi=\left[\begin{array}{c}
\xi_{1} \\
\xi_{2} \\
\vdots \\
\xi_{n-1} \\
\xi_{n}
\end{array}\right] \quad \text { and } \quad \mathbf{b}=h\left[\begin{array}{c}
f_{1} \\
f_{2} \\
\vdots \\
f_{n-1} \\
f_{n}
\end{array}\right]
$$

Consequently, system (3.5) has an unique solution, because $A$ is a sparse, tridiagonal, symmetric and positive definite square matrix, and therefore it has an inverse matrix. 
Finally, coefficients $\xi_{j}$ are obtained and the approximate solution $u_{h}$, which depends strongly on the the function $f$, is determined. The function $u_{h}$ corresponds to the numerical solution of a BVP (3.1) by the FEM [2].

\section{ADAPTATION OF THE SUGENO INTEGRAL}

Definition 4.1 (Sugeno Integral). [9, 12] Let $f: \Omega \rightarrow[0,1]$ be a function and $\tilde{\mu}$ a normalized fuzzy measure on $\Omega$. The Sugeno integral of $f$ with respect to $\widetilde{\mu}$ is given by

$$
f_{\Omega} f d \widetilde{\mu}=\sup _{\alpha \in[0,1]}[\alpha \wedge \widetilde{H}(\alpha)]
$$

where $\wedge$ is the minimum operator and $\widetilde{H}(\alpha)=\widetilde{\mu}(\{x \in \Omega: f(x) \geq \alpha\})$ is called the level function of $f[3]$.

Theorem 1. [3, 12] Let $f: \Omega \rightarrow[0,1]$ be a function (typically a membership function) and $\tilde{\mu} a$ normalized fuzzy measure on $\Omega$. If

$$
\widetilde{H}(\alpha)=\widetilde{\mu}(\{x \in \Omega: f(x) \geq \alpha\})
$$

has a fixed point $\bar{\alpha}$, then

$$
f_{\Omega} f d \widetilde{\mu}=\bar{\alpha}=\widetilde{H}(\bar{\alpha})
$$

In [1], a theoretical and applied study in fuzzy measures and integrals is presented. It also states that the Sugeno integral can be interpreted geometrically as the side of the greatest inscribed square between the integrated function and the $x$-axis (see Figure $2 b$ ).

For example, we can consider the function $f: \Omega \rightarrow[0,1]$ defined by $f(x)=-4 x^{2}+4 x$. Thus, $f$ is a typical membership function of a fuzzy subset $F$ of $\mathbb{R}$ whose $\alpha$-levels are given by

$$
[F]_{\alpha}=\left\{x \in \mathbb{R}:-4 x^{2}+4 x \geq \alpha\right\}=\left[\frac{1-\sqrt{1-\alpha}}{2}, \frac{1+\sqrt{1-\alpha}}{2}\right] .
$$

If $\mu$ is the usual Lebesgue measure (2.2) on $\Omega=\mathbb{R}$, then the level function $\widetilde{H}(\alpha)$ is

$$
\widetilde{H}(\alpha)=\mu\left([F]_{\alpha}\right)=\frac{1+\sqrt{1-\alpha}}{2}-\frac{1-\sqrt{1-\alpha}}{2}=\sqrt{1-\alpha}
$$

Therefore, according to (4.1), the Sugeno integral can be written as:

$$
f_{\Omega} f d \widetilde{\mu}=\sup _{\alpha \in[0,1]}[\alpha \wedge \sqrt{1-\alpha}] .
$$

Since $\widetilde{H}(\alpha)$ is a decreasing function, we have

$$
f_{\Omega} f d \widetilde{\mu}=\bar{\alpha}=\widetilde{H}(\bar{\alpha})=\frac{-1+\sqrt{5}}{2}
$$


since $\bar{\alpha}=\sqrt{1-\bar{\alpha}} \Longrightarrow \bar{\alpha}=\frac{-1+\sqrt{5}}{2} \approx 0.61803$.

The function $f$, the level function $\widetilde{H}(\alpha)$ and the result obtained by the Sugeno integral are shown in Figure 2a and 2b.
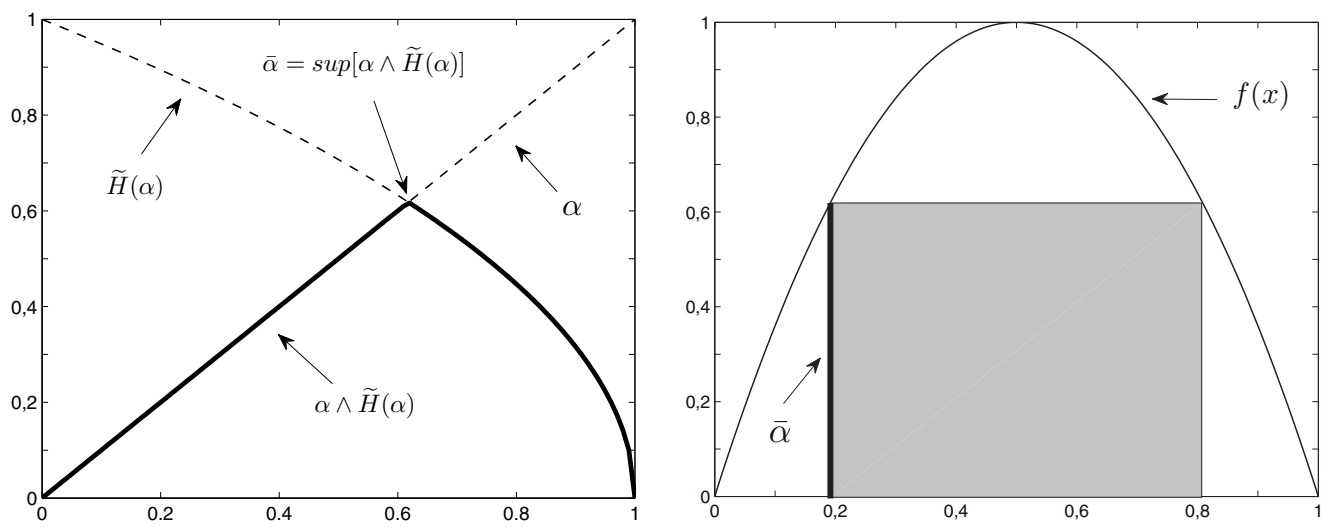

Figure 2: Sugeno integral: (2a) The fixed point of $\widetilde{H}(\alpha)$ and (2b) Geometric interpretation of (4.1).

As mentioned in [7], the Sugeno integral was defined only for functions whose range is contained in $[0,1]$ and for normalized fuzzy measures. As we can note in the previous example, the Sugeno integral is considered a good approximation to the Riemann integral (because $\int_{0}^{1} f d x=2 / 3 \approx 0.66667$ ). However, in [8], it was mentioned that the use of the Sugeno integral for functions whose range is contained in $[0, k], k>0$. But, when $k \neq 1$ or the fuzzy measure $\mu$ is not normalized, the Sugeno integral may not be a good approximation to the Riemman integral (see examples in [10]).

We established an adapted fuzzy integral based on the Sugeno integral and with respect to a finite fuzzy measure. This integral approximates the Riemann integral for any functions whose range is in $[0, k]$, with $k \in \mathbb{R}^{+}$.

Definition 4.2 (Adapted fuzzy integral). Let $f: \Omega \rightarrow[0, k]$ be a measurable function such that $k=\sup _{x \in \Omega} f(x)$. The adapted fuzzy integral of $f$ on $\Omega$, with respect to the finite fuzzy measure $\mu: \mathscr{A} \rightarrow[0, \infty)$, that is, $\mu(\Omega)<\infty$, is given by

$$
\widehat{f}_{\Omega} f d \mu=\left\{\begin{array}{cl}
k \mu(\Omega) f_{\Omega} \widehat{f} d \widetilde{\mu} & , \text { if } k>0 \\
0 & , \text { if } k=0
\end{array}\right.
$$

where $\widehat{f}(x)=\frac{f(x)}{k}$ for all $x \in \Omega$, and $\widetilde{\mu}(B)=\frac{\mu(B)}{\mu(\Omega)}$ for all $B \in \mathscr{A}$.

Remark 1. If $\mu$ is a normalized fuzzy measure and $f$ a function whose range is $[0,1]$, we have that $\mu(\Omega)=1$ and $k=1$. In these conditions, the adapted fuzzy integral (4.2) coincides with the (classical) Sugeno integral (4.1). 
Next, we establish another representation of (4.2) and we present a numerical integration formula to calculate it.

First, from (4.2) and (4.1), we have that

$$
f_{\Omega} \widehat{f} d \widetilde{\mu}=\sup _{\alpha \in[0,1]}[\alpha \wedge \widehat{\widetilde{H}}(\alpha)]
$$

where

$$
\begin{aligned}
\widehat{\widetilde{H}}(\alpha) & =\widetilde{\mu}\{x \in \Omega: \widehat{f}(x) \geq \alpha\}=\widetilde{\mu}\{x \in \Omega: f(x) \geq k \alpha\} \\
& =\frac{\mu\{x \in \Omega: f(x) \geq k \alpha\}}{\mu(\Omega)}=\frac{H(k \alpha)}{\mu(\Omega)}
\end{aligned}
$$

and $H(\beta)=\mu(\{x \in \Omega: f(x) \geq \beta\})$.

Thus, according to Theorem 1 and Equation (4.4), we have that the value of (4.3) is given by

$$
f_{\Omega} \widehat{f} d \widetilde{\mu}=\bar{\alpha}=\widehat{\widetilde{H}}(\bar{\alpha})=\frac{H(k \bar{\alpha})}{\mu(\Omega)} .
$$

Taking $\bar{\beta}=k \bar{\alpha}$, we have that

$$
\bar{\alpha}=\frac{\bar{\beta}}{k}=\frac{H(\bar{\beta})}{\mu(\Omega)} .
$$

Therefore, another representation of (4.2), for $k>0$, is given as

$$
\widehat{f}_{\Omega} f d \mu=k \mu(\Omega) \bar{\alpha}=\mu(\Omega) \bar{\beta}=k H(\bar{\beta}) .
$$

Thus, from Equation (4.5), the calculus of the adapted fuzzy integral boils down to solving the equation $\beta=\frac{k}{\mu(\Omega)} H(\beta)$.

In what follows we provide a numerical integration formula to approximate the Riemann integral, over $[a, b] \subseteq \mathbb{R}^{+}$, of differentiable and increasing functions.

Let $f: \Omega \rightarrow \mathbb{R}^{+}$be a differentiable and increasing function. The Riemann integral of $f$ over $\Omega=[a, b]$ is approximated by

$$
S_{\Omega}(f)=d f(a)+\frac{d c^{2}}{c+d f^{\prime}(b)},
$$

where $c=f(b)-f(a)$ and $d=b-a=\mu(\Omega)$. 
Note that

$$
\begin{aligned}
\int_{a}^{b} f(x) d x & =\int_{a}^{b} f(a) d x+\int_{a}^{b}(f(x)-f(a)) d x \\
& \approx(b-a) f(a)+f_{\Omega} g d \mu \\
& =d f(a)+c \mu(\Omega) f_{\Omega} \widehat{g} d \widetilde{\mu} \\
& \approx d f(a)+\frac{d c^{2}}{c+d f^{\prime}(b)}=S_{\Omega}(f)
\end{aligned}
$$

The value $\widehat{f}_{\Omega} g d \mu=d \bar{\beta} \approx \frac{d c^{2}}{c+d f^{\prime}(b)}$ is obtained as follows. From (4.5), we have

$$
\bar{\beta}=\frac{c H(\bar{\beta})}{\mu(\Omega)}=\frac{c}{d} \mu\left(\left[g^{-1}(\bar{\beta}), b\right]\right)=\frac{c}{d}\left(b-g^{-1}(\bar{\beta})\right)
$$

From $g\left(g^{-1}(\bar{\beta})\right)=g\left(b-\frac{d \bar{\beta}}{c}\right)$ and using first-order Taylor series about $b$, we obtain $\bar{\beta} \approx g(b)-$ $\frac{d \bar{\beta}}{c} g^{\prime}(b)$ and, therefore,

$$
\bar{\beta} \approx \frac{g(b)}{1+\frac{d g^{\prime}(b)}{c}}=\frac{f(b)-f(a)}{\frac{c+d f^{\prime}(b)}{c}}=\frac{c^{2}}{c+d f^{\prime}(b)} .
$$

The next theorem establishes that formula (4.6) coincides with the Riemann integral of certain power functions.

Theorem 2. Let $f: \Omega \rightarrow \mathbb{R}^{+}$be a power function given by $f(x)=c_{1} x^{n}+c_{2}$ with $c_{1}, c_{2}, n \in \mathbb{R}^{+}$, and let $\mu$ be an usual Lebesgue measure in $\Omega=[0, b]$. We have

$$
\int_{0}^{b} f(x) d x=S_{\Omega}(f)
$$

Proof. On the one hand, we have that

$$
\int_{0}^{b} f(x) d x=\int_{0}^{b}\left(c_{1} x^{n}+c_{2}\right) d x=\left.\left(c_{1} \frac{x^{n+1}}{n+1}+c_{2} x\right)\right|_{0} ^{b}=c_{1} \frac{b^{n+1}}{n+1}+c_{2} b .
$$

On the other hand, from (4.6) we get

$$
S_{\Omega}(f)=b f(0)+\frac{b\left(c_{1} b^{n}\right)^{2}}{c_{1} b^{n}+b n c_{1} b^{n-1}}=b c_{2}+\frac{b\left(c_{1} b^{n}\right)^{2}}{c_{1} b^{n}(1+n)}=b c_{2}+c_{1} \frac{b^{n+1}}{1+n} .
$$


A consequence of Theorem 2 for $\Omega=[a, b] \subseteq \mathbb{R}^{+}$is that

$$
\begin{aligned}
\int_{a}^{b}\left(c_{1} x^{n}+c_{2}\right) d x= & \int_{0}^{b}\left(c_{1} x^{n}+c_{2}\right) d x-\int_{0}^{a}\left(c_{1} x^{n}+c_{2}\right) d x \\
= & \underset{[0, b]}{S}\left(c_{1} x^{n}+c_{2}\right)-\underset{[0, a]}{S_{0}\left(c_{1} x^{n}+c_{2}\right)} \\
= & b f(0)+\frac{b(f(b)-f(0))^{2}}{(f(b)-f(0))+b f^{\prime}(b)} \\
& -a f(0)-\frac{a(f(a)-f(0))^{2}}{(f(a)-f(0))+a f^{\prime}(a)} .
\end{aligned}
$$

\section{NUMERICAL APPROACH AND SIMULATIONS}

In the following example, we use the numerical integration formula (4.6) to calculate all the required elements for the matrix system (3.5) and, thus, to approximate the solution of the BVP (3.1) via FEM using an adapted fuzzy integral (4.2) based on the Sugeno integral.

First, in the elements $a_{i, i}$ of the matrix $A$, with $\Omega_{i}=\left[x_{i-1}, x_{i}\right]$, and using equidistant nodes $d=h$ and $c=0$, we have

$$
\begin{aligned}
a_{i i} & =\int_{x_{i-1}}^{x_{i}} \frac{1}{h^{2}} d x+\int_{x_{i}}^{x_{i+1}} \frac{1}{h^{2}} d x \\
& =S_{\Omega_{i}}\left(\frac{1}{h^{2}}\right)+S_{\Omega_{i+1}}\left(\frac{1}{h^{2}}\right)=\frac{1}{h}+\frac{1}{h}=\frac{2}{h} .
\end{aligned}
$$

Thus, we observe that the entries $a_{i i}$ of the matrix $A$ given in (3.8) coincide with those obtained by our proposal in (5.1).

Second, using the adapted fuzzy integral to determine the entries $a_{i, i-1}=a_{i-1, i}$ of matrix $A$, we have $d=h, c=0$, and

$$
a_{i, i-1}=-\int_{x_{i-1}}^{x_{i}} \frac{1}{h^{2}} d x=-S_{\Omega_{i}}\left(\frac{1}{h^{2}}\right)=-\frac{1}{h} .
$$

One can observe that the coefficients $a_{i, i-1}=a_{i-1, i}$ obtained via the classical integration in (3.9) and the our formula (5.2) are equal. By definition of the basis, we have that $a_{i j}=0$ for $|i-j|>1$ since $S_{\Omega_{i}}(0)=0$. 
Finally, the load vector $\mathbf{b}$ is obtained using the numerical integration formula (4.6) for increasing functions $f(x)$ and $g(x)=x f(x)$, for $i=1,2, \ldots, n$ :

$$
\begin{aligned}
b_{i}= & \int_{x_{i-1}}^{x_{i}} f(x)\left(\frac{x-x_{i-1}}{h}\right) d x+\int_{x_{i}}^{x_{i+1}} f(x)\left(\frac{x_{i+1}-x}{h}\right) d x \\
= & \frac{1}{h}\left[\int_{x_{i-1}}^{x_{i}} x f(x) d x-\left(x_{i-1}\right) \int_{x_{i-1}}^{x_{i}} f(x) d x+\left(x_{i+1}\right) \int_{x_{i}}^{x_{i+1}} f(x) d x\right. \\
& \left.\quad-\int_{x_{i}}^{x_{i+1}} x f(x) d x\right] \\
\approx & \frac{1}{h}\left[S_{\Omega_{i}}(g)-\left(x_{i-1}\right) S_{\Omega_{i}}(f)+\left(x_{i+1}\right) S_{\Omega_{i+1}}(f)-S_{\Omega_{i+1}}(g)\right] .
\end{aligned}
$$

In order to illustrate the good results of formula (4.6), we solve the BVP in (3.1) taking the load function $f(x)=15 x^{2} \sqrt{x}$. This yields the exact solution which satisfies the boundary values given by

$$
u(x)=\left(\frac{60}{63}\right)\left(x-x^{9 / 2}\right)
$$
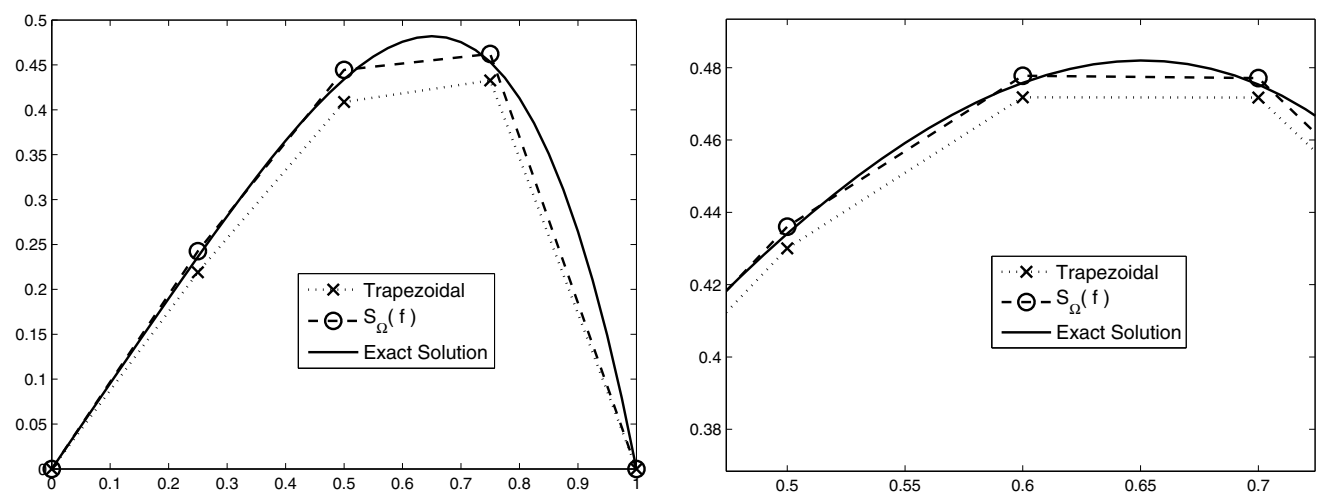

Figure 3: Graphs of the analytic solution for the BVP in (3.1) with $f(x)=15 x^{2} \sqrt{x}$, and the numerical solutions obtained by FEM using the trapezoidal rule and $S_{\Omega}(f)$.

In Figure 3a and Figure 3b, we can see the analytical solution (5.3) as well as the numerical solutions obtained by numerical integration based on the trapezoidal rule (3.10) and by applying the formula (4.6). In this case, for $i=1,2, \ldots, n$, we consider $x_{i}=i h$ to divide the domain $[0,1]$ for both cases.

Table 1 shows the errors $E\left(x_{i}\right)=U_{\text {aprox }}\left(x_{i}\right)-u\left(x_{i}\right)$, for different values of $n$, between the numerical solutions $U_{\text {aprox }}\left(x_{i}\right)$ and the exact solution $u\left(x_{i}\right)$. Here, we consider the infinity norm and the 2-norm [5]:

$$
\|E\|_{\infty}=\max _{1 \leq i \leq n}\left|E\left(x_{i}\right)\right| \text {, and }\|E\|_{2}=\sqrt{\sum_{i=1}^{n}\left|E\left(x_{i}\right)\right|^{2}} .
$$


Table 1: Errors between the numerical solutions and the exact solution of BVP in (3.1), with $f(x)=15 x^{2} \sqrt{x}$.

\begin{tabular}{|c|c|c|c|}
\hline Partition & Integration & $\|E\|_{\infty}$ & $\|E\|_{2}$ \\
\hline \multirow{2}{*}{$\mathrm{n}=3, \mathrm{~h}=1 / 4$} & Trapezoidal & $2.5378 \times 10^{-2}$ & $3.6953 \times 10^{-2}$ \\
& Adap. Fuzzy Int. & $1.0396 \times 10^{-2}$ & $1.5092 \times 10^{-2}$ \\
\hline \multirow{2}{*}{$\mathrm{n}=9, \mathrm{~h}=1 / 10$} & Trapezoidal & $4.0441 \times 10^{-3}$ & $9.3259 \times 10^{-3}$ \\
& Adap. Fuzzy Int. & $1.9478 \times 10^{-3}$ & $4.4831 \times 10^{-3}$ \\
\hline
\end{tabular}

\section{FINAL CONSIDERATIONS}

In this paper, we introduced the notion of an adapted fuzzy integral based on the Sugeno integral. In addition, we propose a numerical integration formula (4.6) for differentiable, increasing, and non-negative functions whose range is contained in $[0, k]$. We verified that, under certain conditions, our proposal yields error equal to zero for power functions, according to Theorem 2. In initial simulations, the numerical solution of a BVP via FEM using our approach obtained better results than a well-known numerical integration method, namely trapezoidal rule (see Figure 3 and Table 1).

\section{ACKNOWLEDGMENT}

This work was partially supported by CONICYT of Chile, CNPq under grant n. 306546/2017-5 and n. 142380/2016-4, and Fapesp under grant n. 2016/26040-7.

RESUMO. Nessa proposta estudamos e definimos uma integral fuzzy adaptada, baseada na integral de Sugeno. Ademais, apresentamos uma fórmula de integração numérica que aproxima o valor da integral fuzzy adaptada. Assim, provamos que a integral de Riemann e a integral fuzzy adaptada são equivalentes para funções potência. Logo, aplicamos a fórmula proposta na integração numérica, requerida no método de elementos finitos, para obter uma solução aproximada de um problema de valor de contorno para a equação de Poisson unidimensional. Finalmente, observamos melhores resultados na solução aproximada obtida com o uso da nossa fórmula quando comparada com a regra simples de trapézio.

Palavras-chave: Medida Fuzzy, Integral de Sugeno, Método de Elementos finitos, Problema de Valor de Contorno.

\section{REFERENCES}

[1] G. Arenas-Diáz \& E.R. Ramírez-Lamus. Medidas difusas e integrales difusas. Universitas Scientiarum, 18 (1)(1) (2013), 7-32.

[2] M. Asadzadeh. An Introduction to the Finite Element Method (FEM) for Differential Equations (2012). URL http://www.math.chalmers.se/ mohammad/teaching/PDEbok/draft_ FEM_version4.pdf. 
[3] L.C. Barros, R.C. Bassanezi \& W.A. Lodwick. A First Course in Fuzzy Logic, Fuzzy Dynamical Systems, and Biomathematics. Springer, New York (2017).

[4] L.T. Gomes, L.C. Barros \& B. Bede. Fuzzy Differential Equations in Various Approaches. SBMAC SpringerBriefs. Springer, New York (2015).

[5] R.J. Leveque. Finite difference methods for ordinary and partial differential equations - steady-state and time-dependent problems. SIAM (2007).

[6] J. Li \& Y. Chen. Computational Partial Differential Equations Using MATLAB. CRC Press Taylor \& Francis Group (2008).

[7] T. Murofushi \& M. Sugeno. Fuzzy measures and fuzzy integrals. Fuzzy Measures and Integrals: Theory and Applications, (2000), 3-41.

[8] H. Nguyen \& E. Walker. A First Course in Fuzzy Logic. CRC Press Taylor \& Francis Group (2006).

[9] W. Pedrycz \& F. Gomide. Fuzzy systems engineering toward human-centric Computing. IEEE Press. John Wiley \& Sons, New Jersey, EUA (2007).

[10] H. Román-Flores, A. Flores-Franulic \& Y. Chalco-Cano. The fuzzy integral for monotone functions. Applied Mathematics and Computation, 185(1) (2007), 492-498.

[11] D. Sánchez, L.T. Bassani, L.C. Barros \& E. Esmi. Ensaios do Método de Elementos Finitos com Integral Fuzzy. In Proceedings of IV CBSF. Recentes Avanços em Sistemas Fuzzy, SBMAC (2016).

[12] M. Sugeno. Theory of fuzzy Integrals and Applications. Ph.D. thesis, Tokyo Institute of Technology (1974).

[13] L.A. Zadeh. Information and control. Fuzzy sets, 8(3) (1965), 338-353.

\section{APPENDIX}

Table 2 presents some numerical results using the numerical integration $S_{\Omega}(f)$ (formula (4.6) based on the adapted fuzzy integral) for differentiable and increasing functions in the interval $\Omega=[0,1]$. The results are compared with others numerical integration rules such as midpoint $I_{M P}(f)$, trapezoidal $I_{\text {Tra }}(f)$ and Simpson $I_{\text {Sim }}(f)$. The reference value was obtained using the Gauss Quadrature rule $I_{G Q}(f) \approx \int_{a}^{b} f(x) d x$, which is an optimal numerical approximation which requires a resolution of a linear system of equations for each integration $[2,5]$.

Table 2: Absolute errors obtained using several rules of numerical integration, and by (4.6).

\begin{tabular}{|c|c|c|c|c|c|}
\hline$f(x)$ & $I_{G Q}(f)$ & $I_{\text {Sim }}(f)$ & $I_{\text {Tra }}(f)$ & $I_{M P}(f)$ & $S_{\Omega}(f)$ \\
\hline$e^{x^{4}}$ & 1.27129 & 1.3294 & 1.8591 & 1.0645 & 1.2345 \\
\hline Error & - & $5.8087 \times 10^{-2}$ & $5.8785 \times 10^{-1}$ & $2.0680 \times 10^{-1}$ & $3.6805 \times 10^{-2}$ \\
\hline$x^{5} \sin (x)$ & 0.12508 & 0.15023 & 0.42074 & 0.014982 & 0.12669 \\
\hline Error & - & $2.5152 \times 10^{-2}$ & $2.9565 \times 10^{-1}$ & $1.1010 \times 10^{-1}$ & $1.6065 \times 10^{-3}$ \\
\hline $15 x^{2} \sqrt{x}$ & 4.2857 & 4.2678 & 7.5000 & 2.6517 & 4.2857 \\
\hline Error & - & $1.7947 \times 10^{-2}$ & 3.2143 & 1.6341 & 0 \\
\hline
\end{tabular}

